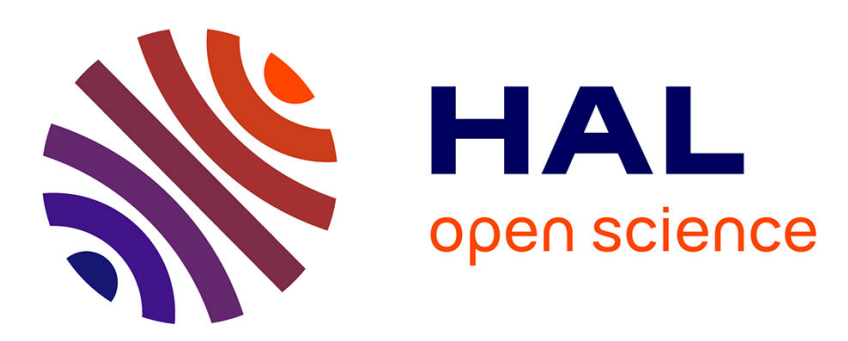

\title{
La sociologie des sciences interrogée par le développement et la diffusion de la méthode diététique du choix du sexe
}

Patrick Trabal, Hélène Mendes, Benjamin Matalon

\section{- To cite this version:}

Patrick Trabal, Hélène Mendes, Benjamin Matalon. La sociologie des sciences interrogée par le développement et la diffusion de la méthode diététique du choix du sexe. Social Science Information, 1999, 38 (3), pp.409-442. 10.1177/053901899038003003 . hal-03016186

\section{HAL Id: hal-03016186 \\ https://hal.parisnanterre.fr/hal-03016186}

Submitted on 20 Nov 2020

HAL is a multi-disciplinary open access archive for the deposit and dissemination of scientific research documents, whether they are published or not. The documents may come from teaching and research institutions in France or abroad, or from public or private research centers.
L'archive ouverte pluridisciplinaire HAL, est destinée au dépôt et à la diffusion de documents scientifiques de niveau recherche, publiés ou non, émanant des établissements d'enseignement et de recherche français ou étrangers, des laboratoires publics ou privés. 
Patrick Trabal, Benjamin Matalon et Hélène Mendes

\section{La sociologie des sciences interrogée par le développement et la diffusion de la méthode diététique du choix du sexe}

Résumé. Le but de cet article est de présenter le cas de la recherche sur l'influence des régimes alimentaires sur le sex-ratio. Il s'agit d'un énoncé émanant d'un scientifique, qui est cependant rejeté par la communauté concernée, tout en étant largement diffusé vers le grand public. Après avoir précisé cette "affaire", nous interrogerons les différents points de vue et modèles prétendant expliquer l'activité scientifique. Nous montrerons que ni une conception scientiste ni le socio-constructivisme ne peuvent prétendre rendre compte finement de ce qui est en jeu dans ce cas. En revanche, une étude sociologique interrogeant l'ensemble des acteurs, leurs logiques, leurs pratiques et les sens qu'ils leur donnent, permet de comprendre cette affaire.

Mot-clés. Biologie - Média - Médecine - Régime - Scientisme - Socio-constructivisme Sociologie des sciences - Vulgarisation scientifique

\section{Introduction}

Dans sa conception idéalisée (et enseignée), le développement de la connaissance scientifique et sa diffusion obéissent à un ensemble de règles, permettant d'une part la production d'énoncés valides et d'autre part le transfert de savoirs utiles pour la société. Le schéma canonique de ce processus tient en quelques concepts clés: démarche hypothético-déductive, priorité aux faits, attitude critique, recherche de consensus au sein de la communauté scientifique. 
Ceux-ci caractérisent le développement de connaissances scientifiques alors que la vulgarisation scientifique a vocation à diffuser des énoncés valides, ce qui est généralement garanti lorsque les scientifiques eux-mêmes s'assurent de la maîtrise de l'information. Certes, face à une telle description du fonctionnement d'une communauté, le sociologue va traquer les écarts entre les règles sociales fixées par les acteurs et leurs pratiques, car précisément ces "déviances" disent quelque chose sur le comportement des acteurs, sur l'évolution des règles sociales que ceux-ci génèrent et donc sur le social.

R. K. Merton (Merton, 1945, 1957, 1973) en essayant de décrire la structure des normes qui guident les scientifiques, insiste sur des normes institutionnelles véhiculées par un système de gratifications symboliques, et notamment sur le contrôle social opéré par la communauté des chercheurs. Cependant l'existence "d'affaires", c'est-à-dire de cas où l'on a transgressé les règles établies, ont conduit certains à critiquer l'institution scientifique, qui serait par ailleurs, autoritaire, conservatrice et avide de pouvoir.

D'autres s'emploient à décrire son fonctionnement comme on le ferait pour n'importe quel groupe social, et affirment qu'il faut renoncer à une vision irénique du fonctionnement de la science pour préférer des modèles sociaux fondés sur des logiques de traduction d'intérêt. Mais cette option est vivement contestée par des sociologues, qui à l'instar de P. Bourdieu (Bourdieu, 1990) affirment que le "champ scientifique" a une spécificité dont l'analyse doit rendre compte: "C'est un monde social comme les autres et comme le champ économique, il connaît des rapports de force et des luttes d'intérêt . . . mais c'est un monde à part, doté de ses propres lois de fonctionnement" (Bourdieu, 1995).

La relation entre l'institution scientifique et la société, quant à elle, n'est que partiellement étudiée. Généralement ceux qui insistent sur la position particulière de l'institution scientifique - le plus souvent des scientifiques - mettent en exergue quelques incompréhensions. Selon eux, le grand public ne comprend ni ce qu'est la science, ni les résultats scientifiques. Ce point apparaît dans la lutte incessante des scientifiques pour lutter contre ce qu'ils considèrent comme des "mauvaises vulgarisations" qui conduiraient certains à utiliser l'aura de la science de façon spécieuse. Les titres et les contenus de quelques communications lors de colloques sont assez significatifs. Nous renvoyons le lecteur avide d'exemples à une série de textes: "Les incompréhensions: l'exemple de la couche d'ozone" (Kandel, 
1991), "Les amours orageuses de la science et de la société" (Hamburger, 1991), "Le dialogue des scientifiques avec les médias" (Kapitza, 1991), "The Prostitution of Science: Pseudo-science" (de Jager, 1991), "Les sciences et les invocations abusives de la Science" (Simon, 1991), "Réflexion sur les sources du malentendu" (Tubiana et al., 1984), "Le paranormal" (Broch, 1985). Et de dénoncer les vulgarisateurs, en invoquant la nécessité de "médiateurs" (Moles et Oulif, 1967; Jacobi et Schiele, 1988) ou en plaidant pour un effort de l'enseignement scientifique (par ex. Tubiana et al., 1984; ANSTJ, 1992).

Des théories comme celles de B. Latour (cf. par ex. Latour, 1987) tentent de ne pas s'intéresser séparément aux deux types de problème que nous venons de rappeler: les écarts entre les normes de production scientifique d'une part et des difficultés de diffusion des connaissances d'autre part. En explicitant le concept de réseau, Latour pense rendre compte de la construction des énoncés scientifiques, qui seraient le résultat de discussions et de négociations gommées par la forme canonique d'un article scientifique, en insistant notamment sur la rhétorique. Il s'agirait de convaincre les scientifiques en recrutant des alliés et en consolidant son réseau. Diffuser un énoncé scientifique dans la société reviendrait à étendre son réseau, c'est-à-dire mobiliser les stratégies nécessaires pour qu'un plus grand nombre d'individus croient en la validité du dit énoncé.

Le propos de cet article est d'interroger ces différents points de vues sur un exemple qui présente l'intérêt de concerner simultanément le fonctionnement de communautés scientifiques et la relation entre l'institution scientifique et la société.

\section{La méthode diététique du choix du sexe}

La méthode du choix du sexe des enfants par un régime constitue un exemple de pratique parfois considérée comme scientifique. Pourtant, en fait, la communauté des biologistes ne la considère pas comme valide. C'est donc un cas intéressant de fonctionnements différents entre trois groupes sociaux: les biologistes, les médecins, et le "grand public". Nous allons tenter de voir comment une affirmation proposée initialement dans un contexte scientifique reconnu, un laboratoire universitaire de biologie, mais rejetée par d'autres chercheurs, s'est néanmoins diffusée et constitue la base d'une technique recommandée par des médecins et des vétérinaires. 
Avant de présenter cette "affaire" de façon plus précise, il convient de préciser ce qui nous a conduit à nous y intéresser et de définir les modalités de notre travail.

Le point de départ de notre recherche est l'intervention d'un chercheur en biologie qui, dans un séminaire de l'Ecole des Hautes Etudes en Sciences Sociales traitant de la sociologie de la connaissance scientifique, a vivement attiré notre attention sur cet exemple: ce cas pose en effet un certain nombre de questions que nous nous proposons de développer ici.

Nous avons alors entrepris une recherche que nous pourrions présenter en trois points. D'une part, nous avons rassemblé un corpus de textes traitant de cette méthode. Nous avons tenté d'élargir au maximum le terrain de nos investigations, puisque les documents recueillis émanent de travaux universitaires, mais aussi de revues médicales, de publications destinées aux vétérinaires, de périodiques de vulgarisation scientifique "grand public" et enfin de la grande presse. D'autre part, nous avons mené une série de neuf entretiens auprès de différents acteurs: des adeptes de cette méthode, des universitaires, des praticiens. Enfin, pour mesurer les effets de la diffusion de cette méthode aujourd'hui, nous avons fait circuler un questionnaire auprès d'étudiants. Parmi les enjeux de cette recherche quantitative, figure une volonté de mieux mesurer les résultats de la diffusion de cette méthode, mais aussi d'étudier les caractéristiques sociales des groupes la connaissant (selon les variables habituelles explorées par la sociologie telles le sexe, l'âge, la spécialisation professionnelle) et enfin d'avoir quelques éléments concernant les conceptions des étudiants sur un sujet qui, à l'heure des manipulations génétiques, se trouve largement médiatisé.

\section{"L'affaire"}

Nous ne reprenons ici que les éléments importants permettant au lecteur de comprendre la discussion: en effet, nous avons dans un premier article montré l'historique de cette recherche sur le sexratio (Trabal et al., 1993). Les grands moments de celle-ci peuvent être regroupés en quatre temps. 
- En 1958, A. Bellec commence une thèse sous la direction du Pr. J. Stolkowski, chercheur en physiologie à la Sorbonne, sur l'étude de l'influence de la teneur en certains ions de l'eau d'élevage de crapauds, sur le sex-ratio. C'était un sujet de recherche assez classique pour l'époque, dans la mesure où l'expression du sexe chez les batraciens est labile et phénotypique. Des premiers résultats furent publiés en s'appuyant sur une courbe qui tente de relier des points assez dispersés (Stolkowski et Bellec, 1959). ${ }^{1}$ Un biochimiste du laboratoire, n'appartenant pas à l'équipe, reprend l'expérience en partant d'eau distillée (et non d'eau de ville) afin de minimiser la dispersion: mais cette dernière fut plus grande (Starer-Mendes et al., 1967). Ceci n'a pas empêché l'auteur de la thèse de la soutenir en 1968, en concluant qu'il y avait une influence de la teneur en ions sur le sex-ratio (Bellec, 1968).

- Stolkowski va essayer de transposer sa problématique à l'influence éventuelle de la teneur en ions ingérés sur des animaux divers, y compris des mammifères. Ceci constitue une certaine rupture dans la recherche, principalement pour deux raisons: d'une part il s'agissait dans la première étude de la teneur en ions dans le milieu de vie, et il s'agit maintenant d'aliments ingérés. D'autre part, contrairement aux batraciens, dans le cas de certains mammifères l'on connaissait les caryotypes et on savait que les expressions génotypique et phénotypique du sexe correspondaient (sauf cas pathologiques). La communauté des chercheurs travaillant sur la reproduction commence par exprimer son scepticisme (Thibault, 1975; Durlach et Pechery, 1977; Courot, 1981) et une équipe de chercheurs réplique l'expérience et obtient des résultats négatifs (Bolet et al., 1982).

- Ne réagissant pas à ces objections, Stolkowski préfère prendre contact avec des médecins et des gynécologues pour étudier les modalités de l'application des premiers résultats au cas humain. Des enquêtes rétrospectives et prospectives sont menées, principalement en France et au Canada, et conduisent les auteurs à affirmer qu'une modification de l'alimentation de la femme avant la procréation peut conduire dans 80 pour cent des cas (voire plus) à obtenir un enfant du sexe désiré (Lorrain et Gagnon, 1975; Stolkowski et Lorrain, 1980).

- Critiqué par la communauté des biologistes, sans néanmoins en être exclu, Stolkowski se tourne alors vers le grand public. Un 
premier article de Science et Vie (Rossion, 1972) affirme la validité de ce qui va devenir une "méthode": Paris-Match, ${ }^{2}$ Le Monde, ${ }^{3}$ la presse médicale,${ }^{4}$ les revues destinées aux vétérinaires et enfin la grande presse spécialisée dans les problèmes de santé ${ }^{5}$ présenteront des taux de réussite assez bons qui justifient la diffusion des modalités pratiques de la méthode. La diffusion se poursuit actuellement: un nouveau magazine consacre cinq pages de son numéro d'avril 1996 à ce sujet (de Hemptine et de Hemptine, 1996); le 8 mai 1996 une émission télévisée, la Marche $d u$ Siècle ${ }^{6}$ mentionnait cette méthode; des vétérinaires achètent encore aujourd'hui des aliments dont la teneur en ions modifierait le sex-ratio. Seul un numéro récent de Science et $\mathrm{Vie}^{7}$ souligne l'existence d'une controverse sur la validité de cette méthode. Par ailleurs, les personnes intéressées peuvent consulter des "spécialistes du sex-ratio"; dans la région parisienne, des consultations sont toujours possibles aux C.H.U. de PortRoyal et Boucicaut. Des médecins proposent aussi des rendezvous dans leur cabinet privé. ${ }^{8} \mathrm{~A}$ l'étranger, le phénomène apparaît comme semblable: à Bruxelles comme à Montréal des hôpitaux proposent des consultations concernant l'application de cette méthode.

Attardons-nous sur les résultats de notre enquête par questionnaire, afin de préciser la réputation de cette méthode aujourd'hui.

\section{Le public et sa connaissance de la méthode}

Nous avons interrogé 227 étudiants qui nous étaient facilement accessibles puisqu'ils fréquentaient les universités dans lesquels nous enseignions en 1997. Notre population d'étude ${ }^{9}$ se répartit en trois groupes d'individus (tableau 1). Quand nous interrogeons l'ensemble de notre groupe sur les possibilités de choisir le sexe de son enfant, 45 pour cent répondent par la négative. Les autres citent souvent un ensemble de méthodes fondées sur des "manipulations génétiques" ou des tris de spermatozoïdes. La méthode diététique n'est mentionnée que dans moins de 10 pour cent des cas.

En revanche, lorsque l'on demande s'ils ont entendu parler de la méthode "des régimes", 65 pour cent répondent par l'affirmative. 
TABLEAU 1

Répartition de la population interrogée

\begin{tabular}{|c|c|c|c|}
\hline & Hommes & Femmes & Total \\
\hline Etudiants en DEUG de biologie à Paris 6 & 34 & 39 & 73 \\
\hline & 13 & 90 & 103 \\
\hline $\begin{array}{l}\text { Etudiants de biologie à Paris } 6 \text { ("Université } \\
\text { dans la Cité") }\end{array}$ & 19 & 32 & 51 \\
\hline Total & 66 & 161 & 227 \\
\hline
\end{tabular}

Mais ils ne la considèrent pas forcément efficace: 60 pour cent des individus interrogés considèrent l'effet du régime sur le sexe de l'enfant comme nul, alors qu'un peu de plus de 35 pour cent lui reconnaissent une influence, souvent considérée comme "réelle mais pas très forte".

Si l'on répartit notre population en deux classes d'âge (25 ans et moins/plus de 25 ans, ce qui conduit à considérer respectivement un groupe de 161 et un de 66 personnes), l'on note que les plus jeunes pensent plus souvent qu'il est possible de choisir le sexe de son enfant, alors que les plus âgés, proportionnellement, sont plus nombreux à considérer l'influence d'un régime comme possible pour avoir un enfant du sexe voulu.

En revanche, les femmes et les hommes ne se distinguent pas de façon significative dans leurs réponses à ces questions (nous sommes très proche du cas d'indépendance).

Enfin, si l'on croise ces variables avec les études poursuivies, on obtient les résultats montrés en tableau 2.

TABLEAU 2

Connaissance de la méthode et opinion la concernant selon le groupe d'appartenance

\begin{tabular}{llll}
\hline & $\begin{array}{l}\text { DEUG } \\
\text { Paris 6 } \\
\text { (biologie) } \\
n=73\end{array}$ & $\begin{array}{l}\text { DEUG } \\
\text { Paris 8 } \\
\text { (psychologie) } \\
n=103\end{array}$ & $\begin{array}{l}\text { "Univ. dans la } \\
\text { Cité" (Paris 6) } \\
\text { (biologie) } \\
n=51\end{array}$ \\
\hline $\begin{array}{l}\text { Ont entendu parler de la } \\
\text { méthode diététique }\end{array}$ & $63 \%$ & $66 \%$ & $65 \%$ \\
La considèrent efficace & $19 \%$ & $22 \%$ & $33 \%$ \\
\hline
\end{tabular}


Ces résultats montrent que les étudiants suivant les cours "université dans la cité" sont proportionnellement plus nombreux à reconnaître une efficacité à cette méthode. Ceci est sans doute dû au fait qu'ils sont plus âgés que les autres, et que par conséquent ils ont eu plus d'occasion d'avoir entendu parler de cette méthode, surtout au moment des premières diffusions au grand public. Notons que les autres étudiants se distinguent peu: ceux évoluant dans des cours de biologie seraient un tout petit peu plus sceptiques envers des affirmations ne figurant pas parmi leurs enseignements ou moins enclins à s'intéresser à des sujets que la biologie semble avoir écarté de son corpus de connaissances, mais l'on reste proche de la situation d'indépendance.

Quoiqu'il en soit, la large diffusion de cette méthode contraste, même si beaucoup ne la considèrent pas comme valide, avec la réaction de la communauté scientifique concernée qui l'a rejetée sans sanctionner explicitement son auteur. Cette affaire interroge donc les modèles prétendant décrire le fonctionnement de la science. Dans un souci dialectique, nous les présenterons en durcissant légèrement la position de leur promoteurs.

\section{Une lecture scientiste ${ }^{11}$ de l'affaire}

Quelle serait l'attitude d'un scientiste à qui on présenterait cette "affaire" ? Invoquant les règles qui fondent le "scientifique" en le distinguant du même coup du "non scientifique", sa lecture attentive l'encouragerait à voir en Stolkowski un personnage qui appartient à la communauté scientifique (il en possède les titres: agrégé, docteur ès sciences, professeur d'université) mais qui s'est écarté, sur ce sujet, de la rigueur et de la discipline qu'impose la science. Si ses autres travaux, la qualité de son enseignement et sa participation à la vie universitaire (membre de commissions, de jurys de thèse) invitent à le considérer comme un scientifique comme les autres, son attitude dans cette "affaire" est condamnable à plus d'un titre. En effet, ses travaux sur le sex-ratio posent plusieurs problèmes.

- Parmi les règles en vigueur dans une communauté d'universitaires (ici celle des physiologistes), certaines concernent la forme de la communication. Il semblerait que les premiers résultats publiés dans le Bulletin de l'Académie des Sciences - de l'équipe du sex-ratio n'obéissent pas aux normes habituellement 
reconnues dans la communauté: en dehors de la fiabilité des points, le fait de tracer une courbe pose problème compte tenu de la dispersion. ${ }^{12}$ Afin de ne pas commettre d'anachronismes, nous avons parcouru d'autres publications de l'époque afin de savoir si cette méthode d'interpolation était usitée. Nous avons en effet trouvé quelques publications dans lesquelles les résultats se présentaient sous forme de segments de droites (Courgeon, 1970) ou d'une courbe passant au milieu de points dispersés (Caussanel et Dresco-Derouet, 1972; Caussanel, 1973) mais les interprétations ne mentionnaient que la forme générale de la courbe et une recherche d'extremum. L'on peut donc affirmer que les résultats présentés par l'équipe de Stolkowski auraient gagné à être présentés avec plus de prudence pour être acceptés par la communauté. Par ailleurs, dès 1967 (Stolkowski, 1967), le rapport des ions est exprimé en termes de force 1, 2 ou 3, ce qui est inhabituel en physiologie. Enfin dans la thèse de DucCabut (Duc, 1977) sur une étude chez la femme, l'on note "+", " ++ ", ou " +++ " la "manière de saler" des femmes. Cette présentation semble donc un indice qui nous conduit à penser que la communauté des physiologistes ne pouvait que marginaliser les recherches sur cette question.

- Si des doutes provenaient du caractère formel de la présentation des travaux, les résultats empiriques restaient faibles. Concernant les recherches sur le crapaud, les conditions d'obtention de ces valeurs posent problème. Les valeurs ioniques ne sont pas constantes car les animaux sont nourris toutes les semaines de salade bouillie dans l'eau du robinet et de viande hachée du commerce; les aquariums n'ont pas tous la même orientation par rapport à la lumière et la température n'est pas constante sur les trois mois que dure le passage de la ponte à l'adulte. Quant à la dispersion des points, il aurait fallu la collaboration de statisticiens pour affirmer l'existence d'une véritable corrélation. Dans toute la recherche sur le sex-ratio, l'on a trace que d'une seule coopération de ce type: elle concerne une étude sur les bovins (Stolkowski, 1984).

Par ailleurs, il aurait fallu s'interroger sur les résultats négatifs afin de parfaire le dispositif expérimental ou de poser des limites aux résultats. En revanche, au lieu d'étudier plus en détail le début de la courbe, l'on passe du batracien au mammifère, sans que l'on soit en mesure d'interpréter les résultats de l'expérience précédente. Une démarche inductive exige un minimum de 
sens critique sur les résultats et une rigueur qui doit permettre de justifier logiquement le recours à une autre expérimentation: l'énorme différence biologique dans la différenciation des gonades entre les batraciens et les mammifères ne peut que susciter un scepticisme quant à la rigueur de la démarche scientifique.

- Néanmoins, le fondement scientifique des mécanismes avancés posait un problème plus grave. Un ensemble de connaissances partagées par la communauté des biologistes était évacué: si l'on se réfère aux premiers mécanismes proposés, il semblerait que les travaux furent entrepris avec l'idée selon laquelle les ions allaient s'intercaler dans les chromosomes et modifier leur déterminisme, ce qui revient à nier - même s'il s'en défend - la théorie syngamique ${ }^{13}$ du déterminisme sexuel.

Cependant en 1972 Stolkowski (Rossion, 1972) a proposé une autre théorie qualifiée de "fécondation préférentielle due aux ions" puis de "sensibilité ovulaire" aux ions, moins éloignée des conceptions de ses collègues physiologistes, qui cependant ne les convainc pas.

- Par ailleurs, son attitude semble exaspérer ses collègues. Il s'entête, ne conserve que les résultats qui vont dans le sens de son propos, ignore les autres, n'écoute pas les conseils l'invitant à renoncer, même lorsqu'ils sont motivés et argumentés par des expérimentations. On pourrait mobiliser de nombreux exemples justifiant ces sévères accusations:

- Un travail sur le caryotype de cellules de rat en culture, qu'il avait commandé à un pédiatre préparant un doctorat de biologie, pour pallier l'impossibilité d'effectuer des caryotypes de batraciens, fera l'objet d'une publication et d'une thèse de troisième cycle (Sebaoun, 1966; Sebaoun et Stolkowski, 1967) et conclura négativement; cependant, seul le matériel d'étude sera incriminé.

- Devant la vigueur des critiques, l'INRA (comme nous l'avons déjà signalé plus haut) a accepté d'arbitrer la controverse mais les résultats furent négatifs et publiés (Bolet et al., 1982). ${ }^{14}$

Ceci n'a en rien arrêté Stolkowski et certains de ses collaborateurs.

- Enfin, généralement les communautés scientifiques s'efforcent de maintenir les controverses à l'intérieur du monde universitaire (Boudon, 1981). Les règles visant à établir des énoncés valides n'ont pas cours en dehors du groupe des scientifiques: ce sont 
d'ailleurs précisément celles-ci qui font le groupe. Elles sont mal comprises à l'extérieur de celui-ci. En diffusant habilement des énoncés que la communauté scientifique concernée juge spécieux, Stolkowski porte la polémique sur la place publique, ce que ses collègues s'interdisent de faire. Par son action, il contribue à des "malentendus", comme le diraient certains (Tubiana et al., 1984): on confond énoncés scientifiques et vulgarisation scientifique, science et "pseudo-science".

Le scientiste, qui se plaît à insister sur ce que la science doit être en définissant les conditions de validité d'un énoncé scientifique et les conditions permettant de les construire, jugera en terme sévère cette "affaire". Les énoncés de Stolkowski sont "non scientifiques" et son attitude est regrettable car une partie du public ( 35 pour cent de la population que nous avons interrogée) croit des choses fausses.

Cette conception de la science, sans doute largement partagée notamment par les biologistes connaissant ce dossier - même si elle est souvent moins tranchée que celle que nous venons de présenter - appelle cependant des critiques.

Avant tout, la notion de validité pose ici problème. Elle fait référence à une réalité qui existerait indépendamment des acteurs humains et des conditions de la recherche scientifique. En fait, nous ne savons pas répondre aux questions suivantes: la cause première des variations du sex-ratio chez les batraciens est-elle due ou non aux modifications de transport ioniques? Le fait qu'une femme suive un régime hyper-potassique ou hyper-calcique a-t-il une influence sur ses chances d'obtenir un enfant du sexe désiré? Nous ne le savons pas. En fait, mis à part Stolkowski et son équipe, on pourrait dire qu'il semble y avoir un consensus dans la communauté scientifique pour dire que l'on ne peut pas répondre à ce jour par l'affirmative. Mais il ne semble pas davantage possible de répondre par la négative. Aucune interprétation ne s'attache à expliquer la forme du nuage de points publié. Tant que l'on ne sait pas évaluer précisément si une femme a suivi fidèlement le régime qu'elle prétend observer, et donc, tant que l'on ne sait pas mettre au point un dispositif expérimental satisfaisant, l'on ne pourra trancher la question. Dans ces conditions, peut-on s'affliger du fait que des gens croient des "choses fausses?" Ainsi, il semble opportun de respecter le principe de symétrie: examiner une controverse en ne se limitant pas à la position d'un seul acteur. 
Par ailleurs, il convient de s'interroger sur la place du social dans l'activité scientifique. Dans la conception précédente, la position institutionnelle de Stolkowski, mais aussi son ambition qui l'a poussé à faire sortir la controverse de la communauté scientifique directement concernée en prenant le grand public à témoin, sont des éléments sociaux qui sont responsables de cette "affaire". Mais nous savons que certains renversent cette problématique en faisant du social, non pas un facteur qui dénaturerait une activité scientifique "pure", laquelle produirait des énoncés dont la validité serait atemporelle, désocialisée, désincarnée, voire transcendante, mais un facteur constitutif de la science. Confrontons donc cette his-toire au modèle socio-constructiviste développé par les promoteurs du programme fort.

\section{L'interprétation par le "social"}

Attardons-nous donc à recenser les conditions sociales qui pourraient expliquer le déroulement de ces recherches. Comment pourrons-nous "pister" et "traquer" - comme B. Latour" ${ }^{15}$ se plaît à décrire son activité - ce qui se jouait dans le développement de la pensée et des stratégies de Stolkowski?

Une méthode conseillée par les promoteurs du programme fort consiste en une analyse de l'ensemble des textes, "au coeur des articles" (Latour et Fabbri, 1977). Nous avons donc rassemblé un ensemble de documents, parmi lesquels les textes scientifiques qui ont fait de Stolkowski un scientifique, l'ensemble des productions universitaires de l'équipe, mais aussi les articles de vulgarisation des-tinés tant au grand public qu'à des lecteurs plus ciblés (les médecins, les vétérinaires) et les ouvrages autobiographiques de l'intéressé. $\mathrm{Si}$ nous restons conscients que ces documents ne peuvent pas être mis sur le même plan, ils nous fournissent cependant un ensemble d'informations qui se recoupent souvent, qui se contredisent parfois, qui dans tous les cas nous éclairent sur les conditions sociales du développement de la méthode diététique du choix du sexe. Ainsi, alors que dans la perspective que nous recensions dans le paragraphe précédent, les énoncés étaient considérés comme statiques et étaient appelés à être évalués sur une échelle dichotomique (scientifique/non scientifique), ${ }^{16}$ nous pouvons maintenant montrer leur origine, leur construction sous la pression de la communauté scientifique, l'évolution de leur forme pour convaincre d'autres publics. 
Le point de départ de la réflexion de Stolkowski provient de l'enseignement qu'il a reçu, enseignement encore très inspiré de lamarckisme $^{17}$ et marqué, comme pour tous ses contemporains zoologistes, par les ouvrages d'E. Wolff: dans son dernier ouvrage, "Les changements de sexe" (Wolff, 1946), cet auteur mentionne l'existence éventuelle de "substances géniques" agissant tant sur les chromosomes autosomes que sur les chromosomes sexuels. Dans ces conditions, Stolkowski, plus proche de la physiologie que de la génétique, pense que certains ions $\left(\mathrm{K}^{+}\right.$et $\left.\mathrm{Ca}^{++}\right)$, dont il connaît bien certains aspects du métabolisme à cause de son travail de thèse (Stolkowski, 1950), pourraient être ces "substances géniques". Cette idée est cohérente avec des résultats publiés en 1932 (Herbst, 1932) sur l'influence de la teneur en ions de l'eau sur le sex-ratio de certains invertébrés.

Les travaux sur les crapauds, donnés comme sujet de thèse, ont été conçus pour étudier un mécanisme éventuel d'intervention des ions agissant directement sur le "matériel chromosomique" - comme l'on disait à l'époque. La conclusion de ce travail mentionne que sans remettre en cause la théorie syngamique du déterminisme sexuel, il est permis d'envisager que les ions $\mathrm{K}^{+}$et $\mathrm{Ca}^{++}$exercent leur action au niveau des chromosomes tant sexuels qu'autosomes. Des dosages prouvant que les ions pénètrent dans le noyau des cellules tendent à confirmer cette thèse. L'idée de Stolkowski reste cohérente avec l'idée que des ions influencent le fonctionnement des chromosomes chez les batraciens, et dans cette perspective le sex-ratio en serait modifié et l'on pourrait agir sur lui; ${ }^{18}$ néanmoins, elle apparaît d'une certaine manière en décalage avec les connaissances génétiques de l'époque. En effet, tout en affirmant qu'il ne niait pas la théorie syngamique (à laquelle la communauté scientifique de l'époque s'était ralliée), il fonctionnait selon un mode de pensée épigéniste (il pensait que les ions allaient s'intercaler dans les chromosomes et modifier leur déterminisme). La pression de la communauté scientifique et l'insistance de ses collaborateurs l'ont probablement amené à taire son intime conviction ou à changer d'avis.

Il évoque alors un mécanisme tout à fait différent. A partir de 1972, dans l'article de Science et Vie (Rossion, 1972), l'on parle de "fécondation préférentielle liée aux ions". Ceci signifie que Stolkowski renonce à l'idée selon laquelle les ions modifieraient les chromosomes, pour affirmer que les spermatozoïdes seraient triés par les ovules sensibles aux ions: le sex-ratio serait donc modifié - 
et donc modifiable - car lié au résultat d'une sélection de spermatozoïdes due à la teneur en ions du "milieu intérieur" (Stolkowski, 1991: 178). Un article d'histologie publié en 1981 (Stolkowski et al., 1981) prouvant que des rates gavées avec de très hautes doses d'ions $\mathrm{Ca}^{++}$voyaient leurs ovaires modifiés, constituera l'argument majeur mentionné dans ses livres et dans des émissions radiophoniques, argument utilisé pour affirmer que les ions agissent sur les gonades, puis alors sur la fécondation. Cette argumentation suffisait à convaincre, des collaborateurs scientifiques (zoologistes, vétéri-naires, médecins) qui appartenaient à une population non sensi-bilisée aux récentes découvertes de la biologie moléculaire, et des auditeurs étrangers à la discontinuité importante apportée dans la pensée biologique par cette nouvelle science.

Il est intéressant d'étudier comment Stolkowski raconte au grand public l'évolution de cette recherche. Son dernier livre (Stolkowski, 1991 ), "Une aventure scientifique", annonce tant par le titre, que par ses premières pages, une volonté de réflexion sur le fonctionnement de l'activité scientifique: "Ce livre a l'ambition de montrer que la Recherche scientifique n'est pas forcément une activité purement intellectuelle, isolée de la vie courante" (p. 9). En clair, il s'agit de mettre en évidence l'importance des facteurs humains et sociaux dans le développement de la science. Le style simple, accessible, volontairement en décalage avec la publication scientifique, présente au non-spécialiste une vie d'un chercheur avec ses doutes, ses problèmes institutionnels, ses joies. Il présente la communauté scien-tifique comme une institution dont l'inertie émane d'un con-servatisme qui ne peut que s'opposer à un esprit libre soucieux d'avancer vers le progrès de l'humanité, ce qui lui permet de décrire son travail en assimilant la validité de sa méthode au progrès et à la vérité qui éclateront tôt ou tard, car il ne peut pas en être autrement. "Certes je pensais à l'Homme, mais incidemment . . . et l'idée m'apparut relever de la Science-fiction en ce sens que j'en concevais

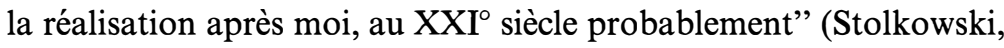
1991: 57).

Ainsi, cette manière de présenter son travail lui permet de se présenter comme un précurseur génial (puisqu'il prétend que le fait est établi au cours de son livre), tout en s'attirant la sympathie du public car il expose une image de la science telle qu'elle permet à des gens d'origine modeste de s'élever socialement (il raconte le début de sa vie), qu'elle contient de l'humain (il raconte ses difficultés à obtenir 
des crédits, des soutiens, de la reconnaissance); le monde scientifique quant à lui est associé à l'idée d'une rigueur et d'un conservatisme excessifs. Or cette rhétorique fonctionne généralement bien: le grand public, lorsqu'il est en position d'arbitrer une controverse entre d'une part un savant isolé et marginalisé et d'autre part l'ensemble de la communauté scientifique, soutient souvent volontiers la cause du "seul contre tous". A des raisons qui mobiliseraient une étude anthropologique, nous préférons invoquer les recherches sur l'image de la science. Construite par les scientifiques eux-mêmes, l'idée selon laquelle le savant peut, à l'instar de Galilée, affronter tous ceux qui ne croient pas aux faits qu'il met en exergue au nom de la vérité, est assez populaire et le fait que Stolkowski, comme Benveniste ou Velikovsky, ${ }^{19}$ communiquent avec le grand public en se présentant comme des génies, victimes du conservatisme de la communauté scientifique, en constitue une illustration. Dans notre cas, le grand public n'était informé de l'existence d'une controverse scientifique sur le sujet, que par le propos de Stolkowski et sa critique du dogmatisme de la communauté scientifique. Cette stratégie fonctionne assez bien, puisque Le Quotidien du Médecin (daté du 27 octobre 1980) titre page 23: "L'imagination n'est pas au pouvoir". Présentant Stolkowski comme le seul qui écouta un paysan constatant la véracité de certains phénomènes en tentant de les expliquer, comme une victime "des milieux officiels, scientifiques ou non" qui préfèrent "l'incrédulité et la répression", comme quelqu'un considéré à tort "comme un 'fou' par des gens 'sérieux", les auteurs de l'article concluent: "Cela rappelle un peu les propos du Pr. Guillemin, Prix Nobel français expatrié aux Etats-Unis, qui a osé dire que la recherche française manquait peut-être d'argent mais aussi, parfois, d'un peu de jeunesse" (Marie et Papa, 1980).

Dans des études sur l'image de la science (Moscovici et Hewstone, 1984; Boy, 1989, 1991; Boy et Michelat, 1993; Trabal, 1995, 1997) cette dernière apparaît souvent comme conservatrice et dogmatique. Par exemple, les chercheurs ayant étudié le succès des parasciences l'interprètent non comme un rejet de la science, mais comme l'expression de la conviction selon laquelle ce qui est parascience aujourd'hui deviendra science demain (Boy et Michelat, 1993). Ceci tend à montrer que le grand public était a priori plutôt prêt à croire les affirmations des chercheurs contestés qui apparaissent généralement comme des précurseurs. Cependant une étude pour comprendre le succès de cette méthode auprès du public reste à faire. 

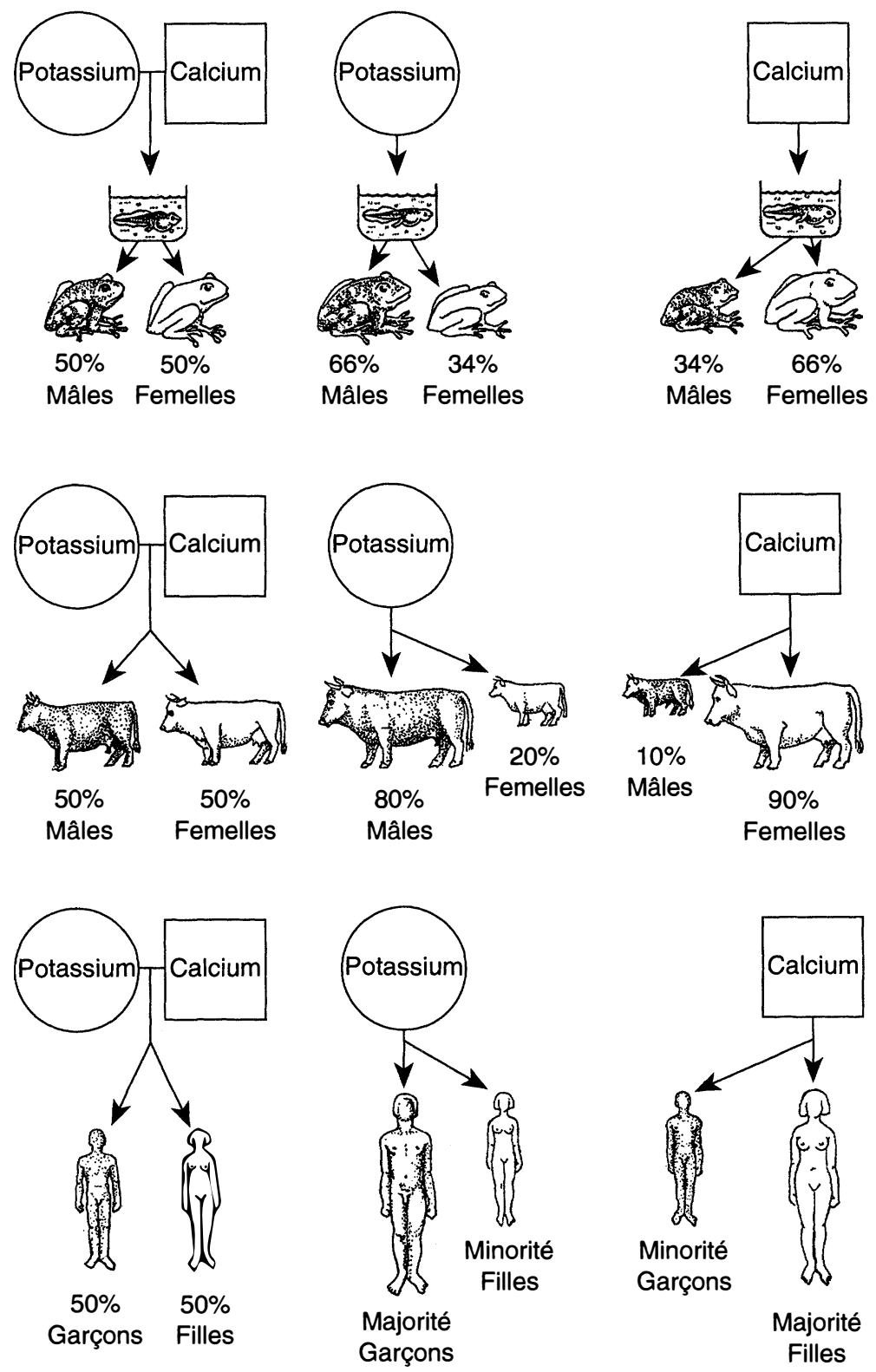

FIGURE 1

Extrait d'un synoptique de Science et Vie (Rossion, 1972) 
Se situant ainsi dans une polémique qu'il raconte en présentant la position de ses adversaires comme des résistances à des faits qui s'imposent, Stolkowski ne peut que constater les signes de sa marginalisation par ses collègues: les refus de publication de ses travaux sur le sujet dès qu'il s'agit de mammifères, les refus de crédits pour ses recherches, les refus de travailler avec lui sont des points que nous analyserons dans le paragraphe suivant et qui ont obligé l'intéressé à se tourner "vers des revues que personne ne lit" (Stolkowski, 1991: 74) puis vers la grande presse (Stolkowski, 1991: 157), à chercher des financements autres et des chercheurs en dehors de la communauté universitaire.

Cette recherche de soutiens - B. Latour parlerait de recrutement d'alliés - le conduit à modifier les énoncés (il modifie sa théorie explicative pour se rapprocher des conceptions plus largement acceptées). En acceptant que Science et Vie publie son travail, il accepte aussi que la forme de son propos évolue (voir figure 1). Comme dans une grande majorité d'articles publiés dans des revues de vulgarisation scientifique, des imprécisions figurent et l'on pourrait expliquer ces dernières comme la contrepartie de la volonté de simplification, obligatoire dans de telles revues: ainsi l'égalité de la proportion de mâles et de femelle constitue une petite erreur, qui ne comporte pas de véritables enjeux. ${ }^{20}$ En revanche, en 1972, l'équipe du sexratio ne disposait pas encore de chiffres pour l'humain. Mais le recours à un synoptique semble suffire pour justifier et valider la transposition de la méthode à l'humain. Au mieux, il s'agit d'une hypothèse. Mais même si l'on accepte la validité des travaux de Stolkowski, l'on ne peut affirmer en 1972, comme le prétend l'auteur de l'article, que l'on "peut influencer la répartition des sexes en agissant sur le régime alimentaire de la mère" - affirmation qui semble valable par déduction sur l'ensemble des espèces présentées sur le document.

Enfin, une dernière remarque s'impose sur ce document: si les chiffres donnés pour le crapaud peuvent être considérés comme exacts (Stolkowski, 1960; Bellec, 1968), ceux avancés concernant la vache n'ont jamais été publié dans les revues scientifiques. ${ }^{21}$ Ainsi avec des informations de ce type, le public se range assez facilement à la version de Stolkowski: plus on se rapproche de l'espèce humaine, plus les résultats sont encourageants.

Afin de gagner en conviction, il "recrute" des acteurs dont la position est centrale: les médecins et gynécologues. ${ }^{22}$ A partir de 1970, il en parle avec le Dr Choukroun qui obtiendra des résultats en 1973; 
en 1972 c'est le Dr Lorrain qui entreprend des travaux menant à une première publication en 1974 (Stolkowski, 1984; Stolkowski et Choukroun, 1991). Mais c'est sans doute son contact en 1976 avec le Dr Papa qui lui donne la possibilité de confronter ses thèses à l'espèce humaine sur un effectif plus important: d'autres acteurs du monde médical lui apporteront leur soutien. ${ }^{23}$ La presse spécialisée a été convoquée pour diffuser la méthode.

Les médecins s'informent généralement de l'actualité des connaissances grâce à des conférences et des revues spécialisées: notamment, Le Généraliste et Le Concours médical. Etudions ce dernier périodi-que, largement diffusé ${ }^{24}$ auprès des médecins abonnés: les articles publiés émanent de médecins, rédacteurs à la revue qui s'informent auprès de spécialistes universitaires ou, suite à des réunions de presse, de médecins qui voient leurs textes soumis à une commission de spécialistes. Il est très probable que les publications sur le thème qui nous intéresse ont été visées par le laboratoire de Stolkowski. ${ }^{25}$ Le médecin qui parcourt régulièrement cette revue a $\mathrm{pu}$ lire quatre articles sur le sujet.

1. Le premier (Henrion et al., 1975) est le compte-rendu d'un forum réunissant Stolkowski et deux gynécologues. Après avoir retracé l'historique de la recherche, ces deux personnalités se déclarent très intéressées par les perspectives énoncées par Stolkowski, même s'ils sont demandeurs de preuves pour confirmer l'hypothèse. Ils concluent sur le fait que la perspective ouverte par ces travaux est passionnante.

2. Un second (Anonyme, 1984) s'attache à mentionner les difficultés liées à la pratique d'un régime rigoureux. Notant que les contraintes sont importantes, l'auteur préconise la prescription d'une thérapeutique complémentaire: ce point laisse supposer que le principe de la modification du sex-ratio ne pose aucun problème.

3. Un troisième te:te (Baudon, 1986) résume prudemment les résultats qui auraient été obtenus. La seule réserve mentionnée concerne l'utilisation de cette méthode qui si elle ne pose problème dans le cas des maladies héréditaires, soulève des questions éthiques liées à 'l'espace de liberté accordé à l'enfant programmé et au sexe assigné" apparaissant aux yeux de l'auteur comme particulièrement atrophié.

4. Enfin, le dernier article (Jeambrun, 1988) laisse apparaître un doute. Après avoir proposé des résultats chiffrés de deux expériences, l'auteur affirme que "l'efficacité reste discutée". Critiquant 
les valeurs numériques en raison de la taille restreinte de la population (liée notamment à de nombreux abandons), il semble adopter une position de neutralité. Le médecin qui, suite à cette lecture souhaiterait en savoir plus, peut essayer de consulter la bibliographie proposée en fin d'article. Une référence concerne une "communication personnelle", l'autre renvoie à une thèse soutenue à Créteil (Duc, 1977) et est donc difficile à se procurer. La dernière mentionne un article dans une revue canadienne (Lorrain et Gagnon, 1975), très difficilement consultable en France.

Ainsi, si les chercheurs n'ont pas été convaincus malgré la tentative de Stolkowski de proposer des explications théoriques plus acceptables par la communauté des biologistes, sa stratégie de diffusion de ses thèses à l'extérieur du champ scientifique restreint des biologistes de la reproduction, fut plus payante. Cette affaire peut donc être racontée en plaçant le social au centre de cette histoire, comme s'il en était le moteur. L'influence de certaines idées (notamment le lamarckisme) a joué un rôle dans la genèse de la pensée de Stolkowski, qui de par sa formation était peu enclin à s'orienter vers les nouvelles orientations proposées par la biologie moléculaire. La pression de ses collègues universitaires le contraint à modifier les théories explicatives du phénomène qu'il prétend avoir mis en évidence, puis à recruter de nouveaux alliés (vétérinaires, médecins, grand public) en faisant des concessions sur le fond et sur la forme. Ainsi, le social peut expliquer la naissance, les balbutiements et la vie d'une recherche qui présente cependant une caractéristique peu banale: des énoncés d'un scientifique, réfutés par la communauté à laquelle il appartient, ont été largement diffusés en dehors du monde scientifique et sont à l'origine de pratiques considérées comme scientifiques (consultations dans des centres hospitalouniversitaires).

Les méthodes et le modèle de traduction, défendus par les promoteurs du programme fort, apportent un éclairage intéressant de cette affaire et des éléments qui échappaient à la perspective précédente. Cependant, cette théorie pose problème.

Si dans notre critique de la conception scientiste, nous avions réfuté l'idée selon laquelle la nature arbitrerait la controverse en désignant un vainqueur, peut-on, comme le prétend B. Latour, affirmer que "la nature est le résultat du règlement de la controverse" (Latour, 1987)? Dans cette perspective, on peut considérer que la controverse n'est pas terminée. Mais, depuis qu'un article 
de réfutation a été publié, les biologistes de la communauté scienti-fique ne poursuivent plus le débat. Il n'y a donc plus de controverse, faute de combattants. Peut-on alors considérer que la thèse de Stolkowski l'a remporté? On pourrait le prétendre: cette théorie s'est imposée, non pas massivement mais de façon sensible dans certains secteurs, notamment dans le domaine agricole et le monde médical. Mais, que fait-on des critiques de la communauté des biologistes?

Pour parler comme le ferait B. Latour, interrogeons le réseau que cette recherche a constitué. On peut dire qu'il est long, ce qui signifie que cette recherche est largement sortie du laboratoire, et donc témoigne d'un certain pouvoir. Mais l'on peut aussi affirmer qu'il est faible, car au sein du laboratoire la thèse n'a pas convaincu. Si un énoncé peut paraître scientifique car il est construit, accepté et relayé par quelques individus généralement considérés comme scientifiques (Stolkowski, les vétérinaires, les médecins), peut-on nier le fait qu'il n'obéit pas aux règles et aux critères fixés par la communauté scientifique concernée?

Si des facteurs sociaux expliquent l'autorité de la science sur ceux qui n'appartiennent pas à la communauté scientifique concernée, autorité qui semble ici pouvoir fonctionner indépendamment des faits validés par ladite communauté, on ne saurait réduire l'activité scientifique à du social. Il convient donc de montrer comment cette situation a été rendue possible: ceci impose de renoncer simultanément à considérer le social comme une perversion de l'activité scientifique, et à penser que les luttes d'intérêt, les règlements de compte et l'usage de rhétoriques visant à convaincre à tout prix expliqueraient tout.

\section{Le fonctionnement de la communauté scientifique}

Les principes qui fondent l'activité scientifique s'articulent autour d'une discussion sur les thèses publiées, d'une réplication d'expérience pour trancher sur une controverse et d'un ensemble de règles qui préservent la spécificité du champ scientifique. Ces règles qui se sont établies au cours d'un long processus où l'on a tenté de normaliser la production de connaissances, fondent "le scientifique" en le contrôlant socialement et figurent parmi les valeurs que l'enseigne-ment scientifique cherche laborieusement à transmettre (Trabal, 1997). 
C'est bien parce que ces règles existent, qu'elles ont été intégrés et qu'elles fonctionnent comme un habitus, que les chercheurs de la communauté scientifique concernée se sont opposés à Stolkowski, ce qui l'a obligé à choisir d'autres stratégies, parmi lesquelles celle de "recruter" d'autres alliés. Or si l'on s'intéresse à ce que cette affaire a apporté à la sociologie des sciences sans tomber dans un "sociologisme" qui nie la spécificité du champ scientifique, il convient de rendre compte des pratiques des acteurs, et notamment de celles qui sont guidés par les règles qui font le scientifique.

\section{La communauté scientifique}

Commencer une recherche sur les influences de certains ions sur le sex-ratio des crapauds n'a pas suscité de polémique. Il s'agissait d'un sujet qui avait peu de chances d'aboutir à des théories nouvelles puisque l'on ne pouvait pas déterminer les caryotypes: cependant rassembler du matériau expérimental dans un domaine où l'on sait peu de choses fait partie de l'activité des zoologistes de l'époque. Le protocole expérimental, la fiabilité des données, la dispersion des points qui n'a pas empêché les auteurs de tracer une courbe et l'hypothèse théorique avancée ont suscité en revanche de vives critiques. ${ }^{26}$ En effet, cela revient à nier une partie des connaissances partagées par la communauté: on savait que des facteurs comme la température pouvaient avoir une influence sur le sex-ratio et ces paramètres n'étaient pas maîtrisés, les connaissances mathématiques des chercheurs les invitent à ne pas tracer de courbes quand le nuage de points est trop éclaté et ceci ne les a pas empêchés de le faire, la première explication théorique venait contredire le paradigme dans lequel tous les scientifiques se retrouvent depuis les travaux de Morgan en 1923. La transgression de cette règle qui impose de s'appuyer sur les méthodes et les théories partagées par la communauté ne se paie pas très cher. Lorsqu'il s'agit d'un étudiant, on le sanctionne par une mauvaise note; lorsqu'il est question d'un chercheur confirmé, on refuse la publication et l'on essaie de discuter.

Une tentative d'opposition à la poursuite des recherches sur le sujet a été de bloquer les crédits de recherche. L'intéressé dénonce cette stratégie en notant "que la communauté scientifique lui enlève tous ses moyens de travail" (Stolkowski, 1991: 111), assertion qui est reprise dans Le Quotidien du Médecin du 27 octobre 1980. Ce 
point mérite d'être examiné de façon plus précise. Le mode d'attribu-tion des crédits universitaires a été modifié à partir de 1970. C'est ainsi que le financement de l'équipe qui nous intéresse est passé de 1967 à 1980 de 60 000F (attribué par le doyen avant modification) à $20000 \mathrm{~F}$ attribués selon le nouveau système en fonction du nombre de chercheurs, qui diminuait constamment. Etant donné le mode d'attribution des crédits universitaires, simplement liés au nombre de personnes travaillant dans le laboratoire, on pourrait donc croire que les pairs n'avaient pas le moyen de sanctionner par ce biais. Cependant suite à cette modification, les équipes univer-sitaires faisaient appel à des financements extérieurs, qui furent refusés à Stolkowski. Or la composition des commissions chargées de gérer ces fonds et de distribuer les crédits (DGRST, ATP-CNRS) fait apparaître qu'il s'agissait des mêmes personnes qui avant 1970 décidaient des dotations aux différents laboratoires. Dans ces conditions, Stolkowski n'a pas tort lorsqu'il affirme que la communauté scientifique le sanctionne en lui retirant tous ses moyens de travail. Cette manière de procéder de ses pairs est finale-ment un désaveu des recherches entreprises par l'équipe du sex-ratio, qui minimise l'affrontement sans faire pour autant des concessions.

Cette stratégie selon laquelle il faut marquer son opposition aux recherches sur le sujet, sans s'opposer trop violemment à Stolkowski, semble centrale si l'on se propose d'expliquer la relative discrétion de ses adversaires.

En effet si une communication orale dans un congrès de physio-

logie à Lyon donne lieu à une discussion houleuse, ${ }^{27}$ il n'y a cependant pas eu de publication de refutation proprement dite. Ce n'est que pour le symposium sur le magnésium qu'il demeure une preuve des réserves émises (Durlach et Pechery, 1977). Si l'on cherche des réfutations publiques, on ne peut en retenir que trois articles scientifiques (Thibault, 1975; Courot, 1981; Bolet et al., 1982), ainsi qu'une émission télévisée où la méthode fut contestée ("L'avenir du futur", TF1, 24 novembre 1980).

Les collaborateurs qui ne veulent pas travailler sur son idée changent de laboratoire: les enseignants-chercheurs de cette époque ont beaucoup de liberté pour effectuer leurs recherches du moment que cela n'entrave pas leur tâche d'enseignement. Ils renoncent à se consacrer à un sujet qui ne leur paraît pas sain: un universitaire ne perd pas son temps avec "l'astrologie" ${ }^{28}$ Par ailleurs, on est en pleine ascension de la biologie moleculaire et des neurosciences. Les élevages ne sont pas à la mode; on ne choisit 
pas de sujets non valorisants. La controverse est avortée car les adversaires de même rang n'y voient pas d'enjeu, surtout depuis la publication de réfutations; les adversaires de rang inférieur estimaient dangereux pour leur carrière de s'attaquer à un professeur d'université. Enfin, tous les acteurs avaient connaissance de la fragilité de la santé de Stolkowski: se lancer dans une polémique sans gros enjeux, au risque de mettre sa carrière en danger, voire de causer un accident cardiaque au responsable de l'équipe, apparaissait comme une tentative dans laquelle on n'avait rien à gagner et tout à perdre. ${ }^{29}$

La réaction de la communauté obéit donc à deux exigences contradictoires: d'une part, respecter un professeur d'université dont on a besoin pour participer à des commissions et à des jurys de thèse, dont on reconnaît la rigueur et la qualité du jugement, et d'autre part refuser de faire des concessions sur l'objet de la controverse.

Ainsi ce travail sur l'attitude de la communauté scientifique permet simultanément de montrer que pour comprendre cette affaire il faut accepter le rôle du social, mais que sa complexité impose de le faire sous certaines conditions. Il ne s'agit pas de l'invoquer simplement pour critiquer un seul protagoniste. A ce titre, il nous faut reconnaître la valeur heuristique du principe de symétrie de D. Bloor (Bloor, 1976), qui exige d'analyser symétriquement les arguments de chacune des parties en évitant d'évaluer un énoncé avec les procédures d'un seul protagoniste (qui généralement est le vainqueur de la controverse). Mais il ne s'agit pas non plus de réduire toute cette affaire à un jeu de luttes d'intérêt. Dire que les chercheurs qui ont critiqué les recherches de Stolkowski, mobilisé des stratégies pour l'empêcher de continuer ses travaux, renoncé à poursuivre la polémique une fois que celle-ci fut placée hors de la sphère contrôlée directement par la communauté scientifique, ont agi dans des buts d'intérêt personnel et de recherche de pouvoir, c'est ignorer qu'ils visent à protéger avant tout un ensemble de normes fondant leur activité scientifique, et qui selon eux furent transgressées et confrontées aux règles en vigueur dans la "société civile" fonctionnant sur le sens commun. L'on pourrait dire, non pas qu'ils se sont uniquement attachés à imposer leur point de vue de scientifiques, mais qu'ils ont surtout cherché à défendre l'institution scientifique et ses règles, mais dans la limite où leurs actions envers Stolkowski n'étaient point dangereuses pour leur carrière. Or parler de rapports de force ou d'intérêt est une manière de se 
demander quels buts poursuivent les chercheurs. Certes, on peut admettre, et c'est ce que font implicitement les épistémologistes, que le seul but du chercheur est la recherche de la vérité. Mais c'est évidemment un peu court comme explication, et au moins insuffisant. Toutefois, inversement, n'y voir que la recherche de satisfactions personnelles, utilitaires, l'est tout autant. Les intérêts invoqués par les sociologues des sciences sont en fait très divers: ce peut être la visibilité dans la communauté scientifique ou en dehors d'elle, la reconnaissance par les pairs, la recherche de pouvoir ou des intérêts sociaux, ceux d'une classe sociale, par exemple, ou encore des intérêts "épistémiques", comme faire triompher une théorie ou étendre le champ d'explication.

Il convient donc d'étudier le fonctionnement de la communauté scientifique sur cette affaire, en dépassant d'une part une lecture naïve des scientistes, et d'autre part le modèle plutôt réducteur de ceux qui ne pensent la recherche scientifique qu'en la résumant à une activité sociale comme les autres.

\section{L'autorité sçientifique}

Ainsi, la sociologie des sciences ne doit ni tomber dans une lecture d'un social qui pervertirait l'activité scientifique et que l'on ne mobiliserait que dans le cas d'une "affaire", ni nier l'existence de règles et de normes qui fondent le champ scientifique, auxquelles des protagonistes se réfèrent, pour lesquelles ils se battent, dans la mesure où ce combat ne nuit pas à leur statut social et professionnel.

Or si ces normes ont guidé les biologistes hostiles aux travaux de Stolkowski, elles fondent l'autorité scientifique sur laquelle ce dernier s'est appuyé pour diffuser sa thèse en dehors de la communauté. En effet, si les médecins, les vétérinaires, le grand public ont cru à l'efficacité de la méthode diététique du choix du sexe, c'est aussi parmi un ensemble d'autres raisons (Trabal, 1996), parce qu'elle émane d'un professeur d'université qui devait posséder des résultats d'expérimentations positifs et les explications théoriques du phénomène.

Les journalistes souhaitant diffuser des informations sur cette méthode contactent directement les spécialistes: pourquoi ne pas s'adresser directement au chercheur à l'origine de cette méthode, surtout si son titre de professeur des universités garantit son sérieux? 
Ainsi Le Monde, sous la plume du Dr Escoffier-Lambiotte (Escoffier-Lambiotte, 1980), rappelle les principaux résultats en s'attachant à détailler les modalités de la méthode pour conclure que "la nature, qui a su veiller par des moyens résistant jusqu'à présent aux investigations les plus raffinées à un équilibre rigoureux des sexes, est peut-être à la veille de voir élucidé son secret". Car les médecins ont peu de raisons de se douter de la faiblesse des résultats qui fondent cette méthode. Ce point est confirmé par les études menées par des sociologues qui s'intéressent à l'information médicale. Pouchain (Pouchain, 1996) s'interroge sur les médias qui véhiculent l'information jusqu'aux médecins: il note que dans la formation médicale, l'écrit revêt un statut équivalent à celui d'une vérité: "C'est avec un esprit critique peu aiguisé vis-à-vis de l'écrit que le jeune médecin aborde la presse médicale".

Si certains médecins, voire équipes médicales, ont été "séduits" par une technique, simple, inoffensive, émanant d'un professeur d'université, c'est sans doute parce que la démarche de pensée médicale est telle qu'on accepte d'appliquer un traitement sans en comprendre la cause: ${ }^{30}$ il a suffi que ce traitement soit cautionné par un spécialiste appartenant au monde universitaire. ${ }^{31}$ Praticiens, ils n'ont certainement pas le temps, ni peut-être les connaissances nécessaires, pour vérifier toutes les affirmations concernant les traitements qu'ils appliquent. De plus il est peu probable qu'un praticien prenne le temps de faire des statistiques systématiques sur les réussites et les échecs des méthodes qu'il prescrit, qu'il s'agisse de celle des régimes dont il est question ici ou d'un traitement quelconque. Il fait confiance à ce qu'on lui a dit, retient quelques cas frappants, attribue les réussites à la méthode elle-même, trouve des explications ad hoc pour certains échecs ou les attribue au fait que la méthode ne garantit pas 100 pour cent de succès. Il se fait ainsi une opinion qu'il peut estimer fondée sur l'expérience: si un universitaire l'affirme, cela doit être vrai.

Le fonctionnement de cette autorité fait l'objet d'analyses différentes et pas toujours contradictoires. La question de la diffusion des résultats scientifiques par recours à la grande presse éclaire ce point. Le travail de R. Boudon (Boudon, 1981) est dans cette perspective intéressant: il tente de recenser les modalités de la communication entre celui qu'il nomme "l'intellectuel-producteur" et son lecteur. Opposant la communication scientifique selon laquelle le jugement des pairs est le principal critère de la diffusion 
de résultats, à la communication s'adressant plus directement au grand public - qu'il illustre par le cas du romancier, considéré comme idéal-typique au sens weberien - il développe l'idée selon laquelle "l'intellectuel-producteur" peut prendre comme cible de son propos un public constitué à la fois de ses pairs et d'un public plus large, ce qui revient à mettre ces différents groupes en concurrence. Cette stratégie, qu'il illustre avec plusieurs exemples, semble être celle adoptée par Stolkowski, et apparaît comme assez souvent utilisée. Dans le cas français, qu'il considère comme spécifique, il affirme:

Ces produits sont souvent confectionnés de manière à entraîner un effet de courtcircuit de l'évaluation par des pairs. Il serait facile de citer de nombreux cas de livres "scientifiques" dont on apprend par les pages culturelles de la presse intellectuelle, dès leur parution et avant toute évaluation par les pairs, qu'ils sont des "contributions scientifiques" fondamentales ou au contraire des productions mineures. (Boudon, 1981)

Ces stratégies indiquent bien qu'une évaluation scientifique est, selon les critères scientifiques, supérieure: il est moins difficile de publier un article dont les conclusions intéressent le grand public dans la grande presse, d'acquérir une reconnaissance de savant dans un monde de non-savant, que dans un espace où le titre de savant n'est attribué que selon des normes. Dans ces conditions, nous ne sommes pas très loin (sur ce point) des propositions de P. Bourdieu qui affirme que chaque champ produit ses propres normes et son propre fonctionnement. Ce qui reste intéressant, c'est d'analyser comment la science acquiert et assure son autorité et sa légitimité.

\section{Conclusion}

Cet exemple permet au sociologue des sciences de réfléchir au poids du social dans l'activité scientifique. Nous avons vu comment une lecture scientiste, qui brandit le social comme une possible source de perversion d'une activité scientifique qui devrait fonctionner sans, réduit une telle affaire à un dysfonctionnement: le scientiste ne peut alors que le regretter en rêvant d'une science pure, le dénoncer comme un accident extérieur regrettable, tenter de l'oublier en l'interprétant comme un mauvais épisode de la recherche de connaissances scientifiques. Nous avons aussi pu évaluer l'intérêt d'une sociologie des sciences qui met le social au coeur de l'activité 
scientifique: en analysant symétriquement les positions des différents acteurs de la controverse, on perçoit l'intérêt du socioconstructivisme. Mais on en mesure aussi les faiblesses. Elles tiennent principalement au fait que certains souhaitent assimiler l'activité scientifique à une activité sociale comme les autres, en la réduisant à des luttes d'intérêt, à des réseaux, à un jeu de pouvoir. C'est oublier sa spécificité. Les logiques des différents acteurs sont certes composées de recherche de la satisfaction d'intérêts sociaux, de luttes de pouvoir, mais sont aussi structurées par un ensemble de normes, de valeurs, d'intérêts épistémiques et par une volonté de défense de l'institution scientifique. On peut bien sûr ramener ces derniers points aux premiers, en affirmant que le combat pour sauvegarder les valeurs scientifiques dissimule une lutte permettant de garantir les intérêts d'un groupe social. L'on écrase alors la quête de vérité scientifique de Stolkowski, la volonté des scientifiques d'être garants de la vérité, le désir d'informer des vulgarisateurs, d'une façon plus générale le sens que les acteurs sociaux donnent à leurs pratiques en les réduisant à une lutte de pouvoir. On tombe alors dans un réductionnisme primaire.

Cet exemple nous a permis de mettre en exergue deux écueils qui peuvent menacer notre compréhension de la place du social dans l'activité scientifique: la réduction scientiste des facteurs sociaux à un problème externe qui pollue le travail des scientifiques et la réduction des sociologies des sciences constructivistes qui oublient la spécificité du travail des chercheurs et le sens qu'ils leur donnent. Nous pensons cependant avoir montré qu'en mobilisant sociologues et biologistes, il est possible de comprendre ce qui se joue socialement dans une affaire comme celle-ci.

Patrick Trabal (né en 1963) est Maître de conférences à l'Université Paris XNanterre. Il a récemment publié La violence de l'enseignement des mathématiques et des sciences: une autre approche de la sociologie des sciences, Paris: L'Harmattan (1997). Adresse de l'auteur: Université Paris X-Nanterre, Bât. M, 200 av. de la République - F 92001 Nanterre Cedex.

Benjamin Matalon (né en 1930) est professeur de sociologie à l'Université Paris VIII-Saint Denis. Il a récemment publié La construction de la science, Paris: Delachaux et Niestlé (1996). Adresse de l'auteur: Groupe d'Etudes et de Recherches sur la Science (GERS), 10 rue Monsieur le Prince, F 75006 Paris.

Hélène Mendes (née en 1940), Maître de conférences en biologie à l'Université Paris VI-Jussieu, est chargée de recherches sur la communauté scientifique et la bio éthique. Adresse de l'auteur: Laboratoire de Zoologie, Université Paris VI, Bât.A., pièce 514, 11 Quai Saint-Bernard, F 75005 Paris. 


\section{Notes}

1. En annexe (Fig. 2) nous avons reproduit la courbe publiée, puis le nuage de points obtenus.

2. Cf. Serrou (Serrou, 1980).

3. Cf. Escoffier-Lambiotte (Escoffier-Lambiotte, 1980).

4. Voir plus bas.

5. Voir par exemple Malot (1977), de Tallano (1982), Gaudin (1988), Roy (1991) et un article anonyme dans Réponse à Tout - Santé (Anonyme, 1991).

6. L'émission s'intitulait "Bébés sur catalogue: la science jusqu'où?".

7. Le dossier "On peut choisir le sexe de son enfant" du numéro évoque principalement les techniques de sélection des spermatozoïdes. La méthode diététique est mentionnée dans un article recensant tous les essais (Moinet, 1998).

8. Lors de notre enquête téléphonique (en 1996) auprès des différents hôpitaux parisiens, on nous a informé de la possibilité de consultations dans plusieurs cabinets privés.

9. Sur la question de l'opportunité du choix du sexe, on peut se reporter au sondage commandé par Science et Vie et réalisé en septembre 1998 par l'Institut français d'opinion publique; il apparait que les français restent majoritairement hostile au recours à des procédés permettant de choisir le sexe de l'enfant à naître (cf. dossier Science et Vie, 1998). Malgré l'intérêt de cette étude qui porte sur 951 individus, aucune question ne se référait à la méthode diététique.

10. Il s'agit d'un public inter-âge (l'âge moyen est de 65 ans), qui est inscrit à l'université et suit des cours quotidiens donnés par les enseignants des UFR sur la base du volontariat.

11. Ce terme étant connoté souvent péjorativement, il nous faut préciser son acception: est scientiste, dans notre perspective, celui qui croit à la possibilité d'une connaissance objective et à des méthodes adéquates pour y arriver.

12. Lors d'une communication sur cette affaire (lors de la 2ème réunion annuelle de la Société d'Histoire et d'Epistémologie des Sciences de la Vie, Paris 22-23 mars 1996) nous avons présenté la courbe que nous reproduisons en annexe en ayant retiré les points. Dans la salle, essentiellement composée de biologistes, plusieurs personnes ont manifesté leur étonnement: "il faut être 'gonflé' pour oser tracer une courbe làdedans".

13. Selon laquelle le déterminisme sexuel a lieu à l'instant même de la fécondation. Cette théorie s'oppose à la théorie épigamique tombée en désuétude à partir de 1923, après la démonstration de la détermination chromosomique du sexe par T. H. Morgan (Morgan et al., 1923): ceci a entraîné l'abandon progressif de ces deux termes.

14. La publication d'un article présentant des résultats négatifs est assez rare. Il convient de noter que la conclusion de ce texte mentionne le fait que l'hypothèse est réfutée tout en posant la question de l'explication du fait qu'un "mécanisme prétendu général d'action des ions minéraux ... n'agirait pas du tout dans [ces] conditions expérimentales".

15. Si nous nous référons surtout à $\mathrm{B}$. Latour pour développer ce point de vue, en le préférant aux universitaires anglo-saxons qui défendent des thèses assez proches des siennes, c'est parce qu'il développe un modèle peut-être plus complet (comprenant notamment les notions de réseau, de traduction) que l'on ne retrouve pas chez ses collègues. 
16. En fait il est vrai que des chercheurs s'emploient à doser le degré de crédit qu'ils apportent aux énoncés; cependant cette démarche suppose qu'ils considèrent l'énoncé comme recevable scientifiquement. On critique les faiblesses d'un article scientifique (en évaluant le crédit qu'on peut lui apporter pour savoir dans quelles conditions on peut, par exemple, le citer) alors que l'on rejette un texte qui ne mérite pas le qualificatif "scientifique".

17. L'influence du lamarckisme dans la biologie française semble avoir été sensible jusqu'en 1950.

18. Il est sans doute intéressant de préciser qu'en 1997 personne n'est en mesure d'expliquer totalement les modifications phénotypiques de l'expression sexuelle de certains invertébrés et de certains poissons; cependant tout le monde s'accorde pour affirmer la constance du programme génétique de départ, la régulation interviendrait plutôt au niveau transcriptionnel ou traductionnel.

19. Velikovsky était un psychiatre qui sans connaïtre particulièrement les sciences affirmait dans les années 1950 une remise en cause fondamentale de l'histoire récente de la Terre et de l'astronomie. Soutenu par quelques scientifiques, il s'est heurté à une opposition farouche de chercheurs qui ont maladroitement déclaré que cette théorie était tout simplement impossible. Cette "affaire" illustre le dogmatisme de la "science officielle" qui renonce au débat, lorsque l'énoncé apparaît comme aberrant ou incompatible avec ce qui est admis. Voir à ce sujet H. Bauer (Bauer, 1984).

20. S'il est habituel de dire que la répartition de la population est 50 pour cent de mâles et de 50 pour cent de femelles, il s'avère qu'il y a en fait 52 pour cent de mâles, cet écart ne s'expliquant d'ailleurs pas avec les connaissances actuelles.

21. Nous ne retrouvons pas ces valeurs dans l'ensemble des publications sur la vache (Stolkowski, 1966, 1967; Stolkowski et Emmerich, 1969, 1971; Stolkowski et Lefêvre, 1977, 1980).

22. D'autres "recrutements" ont lieu, notamment chez les vétérinaires et les acteurs du monde agricole. Notre premier article (Trabal et al., 1993) précise que de nombreuses expérimentations ont été menées sur des animaux en fonction des opportunités et des individus que Stolkowski a réussi à convaincre. Nous ne détaillons pas le développement de la méthode "des régimes" dans ce champ, afin de mieux analyser sa diffusion dans le monde médical et dans le grand public.

23. C'est ce qu'il affirme dans son livre (Stolkowski, 1991). Nous avons trace également de cette collaboration avec le Dr Papa présent lors de la conférence sur le magnésium (Durlach et Pechery, 1977). Les détails de cette association nous ont été racontés lors des entretiens.

24. En 1996, la revue revendique 24000 lecteurs; afin de donner un ordre d'idée, l'on recensait alors environ 180000 médecins en France.

25. C'est d'ailleurs également le cas pour le concurrent du Concours médical, Le Généraliste, qui reproduit le propos de Stolkowski (Fournier, 1980).

26. Ce point, qui ne figure pas dans les publications, est mentionné plusieurs fois lors des entretiens.

27. Communication personnelle d'un participant.

28. Mot effectivement employé par un collègue de Stolkowski lors d'un entretien.

29. Ce point apparait notamment dans l'entretien que C. Thibault a bien voulu nous accorder, ainsi que dans un témoignage d'un référé.

30. L'on peut mentionner le cas de l'aspirine, dont l'efficacité a conduit pendant longtemps à de nombreuses prescriptions, alors que son mécanisme vient juste d'être partiellement élucidé. Ce mode de fonctionnement ne caractérise pas le monde 
médical: il est celui de nombreux praticiens qui utilisent des résultats "pratiques" qu'un autre groupe social a pour mission d'élaborer.

31. Dans le cas qui nous intéresse, l'universitaire (Stolkowski) n'hésite pas à reconnaître devant un lectorat de médecins qu'il ne peut expliquer le phénomène alors que, nous l'avons vu, il a proposé dans d'autres occasions trois modèles interprétatifs en fonction de son public. Ainsi, dans Le Généraliste, on lit: "Le problème qui intéresse le Pr. Stolkowski, ce n'est pas le mécanisme d'action du déterminisme du sexe "qui n'est pas à la portée du laboratoire dans l'état actuel de nos connaissances", mais c'est de recenser les facteurs qui peuvent interférer avec le processus, de perfectionner le procédé actuellement expérimenté et d'améliorer ainsi le pourcentage de réussite" (Fournier, 1980).

ANNEXE: FIGURE 2

Les variations du sex-ratio chez les batraciens en fonction des modifications de transport ioniques

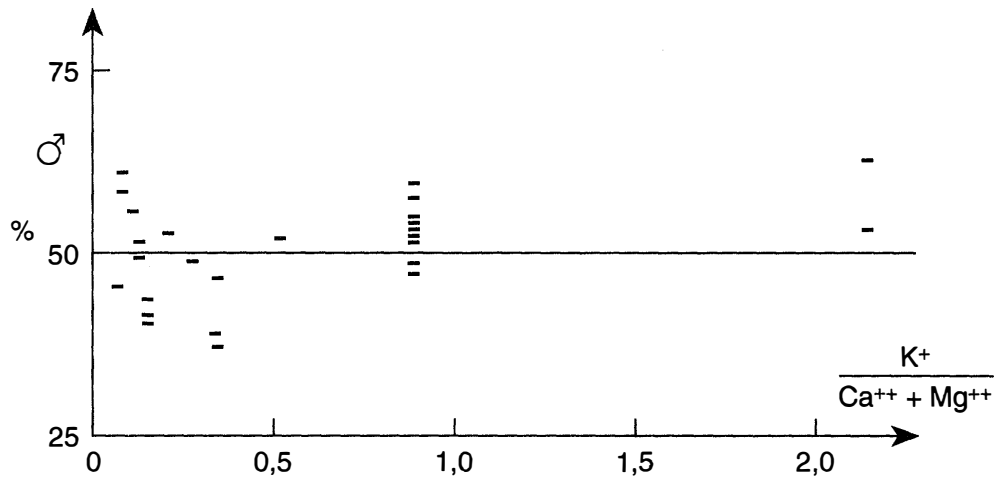

Références

Anonyme (1984) "Sélection du sexe, des méthodes et des résultats intéressants", $L e$ Concours Médical 3878: 106-40.

Anonyme (1991) "Fille ou garçon vous pouvez choisir à coup sûr", Réponse à ToutSanté 3 (décembre).

ANSTJ (Association Nationale Sciences Techniques Jeunesse) (1992) Actions et réactions. Paris: Z'éditions (Collection A. Giordan et J.L. Martinand).

Baudon, D. (1986) "Courrier des lecteurs - pour avoir une fille", Le Concours Médical 1763: 108-21.

Bauer, H. (1984) Beyond Velikovsky: The History of a Public Controversy. Chicago: University of Illinois Press. 
Bellec, A. (1968) "Influence des variations du rapport $(\mathrm{K} / \mathrm{Ca})$ du milieu d'élevage sur le développement de la croissance et la répartition des sexes chez les têtards de Discoglossus pictus (Otth)", thèse, Université Paris VI.

Bloor, D. (1976) Knowledge and Social Imagery. London: Routledge \& Kegan Paul. (Traduction française Sociologie de la logique. Paris: Pandore, 1983.)

Bolet, G., Guéguen, L., Dando, P. et Ollivier, L. (1982) "Influence de l'alimentation minérale de la truie sur le taux de masculinité à la naissance", Reports in Nutritional Development 22 (6): 1073-81.

Boudon, R. (1981) "L'intellectuel et ses publics: la singularité française", in J.D. Reynaud et Y. Grafmeyer (eds) Français qui êtes-vous?, pp. 465-80. Paris: La Documentation Française.

Bourdieu, P. (1990) “Animadversiones in Mertonem", in C. Clark et S. Modgil (eds) Robert K. Merton: Consensus and Controversy, pp. 297-301. London and New York: Falmer Press. (Ce texte a été publié en français "La double rupture", in Raisons pratiques sur la théorie de l'action, pp. 91-7. Paris: Seuil, 1994.)

Bourdieu, P. (1995) "La cause de la science: comment l'histoire sociale des sciences sociales peut servir le progrès de ces sciences”, Actes de la Recherche en Sciences Sociales 106-7: 3-10.

Boy, D. (1989) "Les attitudes des Français à l'égard de la science", in Rapport de résultats et note de synthèse. Paris: F.N.S.P./CEVIPOF (avril).

Boy, D. (1991) "Evolution des attitudes depuis 1972", in Colloque pour la science, pp. 17-33. Paris: Fondation Electricité de France/Cité des Sciences et de l'Industrie (Actes du colloque).

Boy, D. et Michelat, G. (1993) "Premiers résultats de l'enquête sur les croyances aux parasciences", in La pensée scientifique et les parasciences, pp. 209-24. Paris: Albin Michel/Cité des Sciences et de l'Industrie.

Broch, H. (1985) Le paranormal. Paris: Seuil.

Caussanel, C. (1973) "Variations et principales fonctions du corpus allatum au cours des cycles reproducteurs de la femelle de Labidura riparia Palla (insecte, dermaptère)", Comptes Rendus de l'Académie des Sciences, Paris 277: 1521-4.

Caussanel, C. et Dresco-Derouet, L. (1972) "Respiration de la femelle de Labidura riparia insecte dermaptère avant la ponte et pendant la période de soins aux oeufs", Comptes Rendus de l'Académie des Sciences, Paris 274: 1179-82.

Courgeon, A.M. (1970) "Croissance des disques oculo-antennaires de larves de Calliphora erythrocephala Meig (insecte diptère) en culture in vitro", Comptes Rendus de l'Académie des Sciences, Paris 270: 1815-18.

Courot, M. (1981) "Peut-on choisir le sexe des enfants?", La Recherche 12, 119: 218-20.

de Hemptine, D. et de Hemptine, C. (1996) "Le choix du sexe de votre enfant", Naître, Bruxelles 4: 24-8.

de Jager, C. (1991) "The Prostitution of Science: Pseudo-science", in Colloque pour la science, pp. 120-6. Paris: Fondation Electricité de France/Cité des Sciences et de l'Industrie (Actes du colloque).

de Tallano, G. (1982) "Fille ou garçon peut-on choisir?", Santé Magazine 84 (décembre).

Duc, M. (1977) “De l'influence des apports nutritionnels en ions $\mathrm{Na}, \mathrm{K}, \mathrm{Mg}, \mathrm{Ca}$, sur le sex-ratio chez l'homme", thèse de médecine, Créteil. 
Durlach, J. et Pechery, Y.-C. (1977) "Compte rendu du 4ème colloque annuel sur le magnésium", Revue Française d'Endocrinologie Clinique, Nutrition et Métabolisme 18 (2): 123-5.

Escoffier-Lambiotte, C. (1980) "Garçon ou fille à volonté", Le Monde (2 avril).

Fournier, S. (1980) "Déterminisme du sexe des enfants: le rôle des régimes alimentaires", Le Généraliste 320 (8 novembre).

Gaudin, M. (1988) "Grossesse, choix du sexe: de nouveaux progrès", Parents (août).

Hamburger, J. (1991) "Les amours orageuses de la science et de la société", in Colloque pour la science, pp. 108-12. Paris: Fondation Electricité de France/Cité des Sciences et de l'Industrie (Actes du colloque).

Henrion, R., Papiernik, E. et Stolkowski, J. (1975) "Choisir le sexe avant la conception", Le Concours Médical 97-43: 7077-83.

Herbst, C. (1932) "Die Vermännlichung der Larven von Bonnelia viridis durch Kupferspuren", Naturwissenschaft 22: 375-9.

Jacobi, D. et Schiele, B.(1988) Vulgariser la science, le procès de l'ignorance. Seyssel: Champ-Vallon.

Jeambrun, P. (1988) "Sélection préconceptionnelle du sexe par le régime alimentaire", Le Concours Médical 110-35: 3165-7.

Kandel, R. (1991) "Les incompréhensions: l'exemple de la couche d'ozone", in Colloque pour la science, pp. 74-90. Paris: Fondation Electricité de France/Cité des Sciences et de l'Industrie (Actes du colloque).

Kapitza, S. (1991) "Le dialogue des scientifiques avec les médias", in Colloque pour la science, pp. 113-19. Paris: Fondation Electricité de France/Cité des Sciences et de l'Industrie (Actes du colloque).

Latour, B. (1987) La science en action, traduit de l'anglais par M. Biezunski. Paris: La Découverte.

Latour, B. et Fabbri, P. (1977) "La rhétorique de la science: pouvoir et devoir dans un article de science exacte", Actes de la Recherche en Sciences Sociales 13: 81-95.

Lorrain, J. et Gagnon, R. (1975) "Sélection préconceptionnelle du sexe", Union Médicale du Canada 104 (5): 800-3.

Malot, J.M. (1977) "Fille/garçon un choix possible", Santé Magazine 19 (juillet).

Marie, L. et Papa, F. (1980) "Déterminisme préconceptionnel du sexe, les enfants ne sont pas des anges", Le Quotidien du Médecin (27 octobre).

Merton, R.K. (1945) "Sociology of Knowledge", in G. Gurvitch et W.E. Moore (eds) Twentieth Century Sociology, pp. 366-405. New York: Philosophical Library.

Merton, R.K. (1957) Social Theory and Social Structure. Glencoe, IL: The Free Press.

Merton, R.K. (1973) The Sociology of Science. Chicago, IL: University Press of Chicago.

Moinet, M.-L. (1998) "Un rêve vieux comme le monde", Science et Vie 974 (novembre): 71-2.

Moles, A. et Oulif, J.M. (1967) "Le troisième homme, vulgarisation scientifique et radio", Diogène 58: 29-40.

Morgan, T.H., Sturtevant, A.H., Muller, H.J et Bridges, C.H. (1923) Le mécanisme de l'hérédité mendelienne. Bruxelles: Lamertin.

Moscovici, S. et Hewstone, M. (1984) "De la science au sens commun", in S. Moscovici (ed.) Psychologie sociale, pp. 539-66. Paris: PUF.

Pouchain, D. (1996) "Comment l'information vient aux médecins", in E. Galam (ed.) Infiniment médecins, pp. 141-53. Paris: Autrement (Collection Mutations, 161). 
Rossion, P. (1972) “On pourrait vraiment faire fille et garçon à volonté”, Science et Vie 656: 56-61.

Roy, C. (1991) “Peut-on vraiment choisir le sexe de son enfant?", Parents (septembre).

Sebaoun, M. (1966) “Contribution à l'étude de l'influence exercé par les variations de concentration en potassium, calcium, magnésium, du milieu sur les cellules de rat en culture", thèse, Faculté des sciences, Paris.

Sebaoun, M. et Stolkowski, J. (1967) "Etude du comportement et du caryotype de cellules de rat en culture sous l'influence des variations des concentrations ioniques du milieu en potassium, calcium et magnésium", Archives des Sciences Physiologiques 21 (3): 365-79.

Serrou, R. (1980) "Sexe à volonté", Paris-Match (18 avril).

Simon, G. (1991) "Les sciences et les invocations abusives de la Science", in Colloque pour la science, pp. 139-49. Paris: Fondation Electricité de France/Cité des Sciences et de l'Industrie (Actes du colloque).

Starer-Mendes, H., Trombert, M. et Stolkowski, J. (1967) “Action de l'ion Mg sur la répartition des sexes dans une population de têtards élevés en solution minérale chez Discoglossus pictus (Otth)", Comptes Rendus de l'Académie des Sciences, Paris 265: 1518-21.

Stolkowski, J. (1950) "Essai sur le déterminisme des formes minéralogiques du calcaire chez les êtres vivants", thèse, La Sorbonne, Paris.

Stolkowski, J. (1960) "Recherches sur les échanges cellulaires en potassium et leurs incidences biologiques, en particulier sous l'influence d'hormones corticostéroides", in Potassium Symposium. Berne: Institut International de la Potasse.

Stolkowski, J. (1966) “Recherches sur l'action biologique de certains éléments minéraux de la ration alimentaire (potassium calcium magnésium) chez les animaux d'élevage, vaches moutons poulets", in 9lème Congrès des Sociétés Savantes, pp. 275-80. Rennes: Gauthier-Villars.

Stolkowski, J. (1967) "Influence possible de la nutrition minérale sur la répartition des sexes chez la vache: une enquête rétrospective", Comptes Rendus de l'Académie des Sciences, Paris 265: 1059-62.

Stolkowski, J. (1984) Le secret du paradis: choisir le sexe de son enfant par une alimentation appropriée. Montréal: Les Editions de L'Homme.

Stolkowski, J. (1991) Une aventure scientifique, le choix du sexe. Paris: Chiron.

Stolkowski, J. et Bellec, A. (1959) "Influence des rapports ioniques du milieu sur la croissance et le développement des embryons de Discoglossus pictus (Otth) et Rana temporaria (Linné). Premières observations", Comptes Rendus de l'Académie des Sciences, Paris 248: 3349-51.

Stolkowski, J. et Choukroun, J. (1991) Garcon ou fille, le choix, par sélection préconceptionnelle: la méthode naturelle du Professeur Stolkowski. Paris: Chiron.

Stolkowski, J. et Emmerich, E. (1969) "Influence de la nutrition minérale sur la répartition des sexes chez la vache, une enquête prospective, un essai expérimental", Comptes Rendus de l'Académie des Sciences, Paris 268: 2787-90.

Stolkowski, J. et Emmerich, E. (1971) "Influence de la nutrition minérale de la vache sur la répartition des sexes dans la descendance: une enquête prospective, un essai expérimental", Annales d'Endocrinologie de Paris 32 (1): 3-15.

Stolkowski, J. et Lefêvre, M. (1977) "Essai de contrôle du sexe chez les bovins sous l'influence de la nutrition minérale", Recherches en Médecine Vétérinaire 153 (1): 37-44. 
Stolkowski, J. et Lefêvre, M. (1980) "L'excès de calcium et de vitamine D3 pourrait augmenter le nombre de femelles dans la descendance", Production Laitière Moderne 83: 15-18.

Stolkowski, J. et Lorrain, J. (1980) "Preconceptional Selection of Fetal Sex", International Journal of Gynecology and Obstetrics 18: 440-3.

Stolkowski, J., Laroche, E. et Vovelle, J. (1981) "Calcium ovarien et fécondation chez la rate recevant une surcharge subchronique en vitamine D", Comptes Rendus de la Société de Biologie 175: 152-9.

Thibault, C. (1975) "La fécondation", in Colloque de la Société nationale pour l'étude de la stérilité et de la fécondation, pp. 94-136. Paris: Masson.

Trabal, P. (1995) "Le sens commun face à ia science: vers l'étude des représentations de la science", Revue des Questions Scientifiques, Bruxelles 166 (1): 39-54.

Trabal, P. (1996) "Eléments historiques et sociologiques concernant la diffusion de la méthode diététique du choix du sexe", Médecine/Science 12, 11: 1293-6.

Trabal, P. (1997) La violence de l'enseignement des mathématiques et des sciences une autre approche de la sociologie des sciences. Paris: L'Harmattan (Collection Education et Formation - série Références).

Trabal, P., Mendès, H. et Matalon, B. (1993) "La communauté scientifique face à la méthode diététique du choix du sexe", Revue des Questions Scientifiques, Bruxelles 164 (3): 269-91.

Tubiana, M. (1984) "Réflexion sur les sources du malentendu", in M. Tubiana, Y. Pelicier et A. Jacquard (eds) Image de la science. Paris: Economica (Actes du colloque).

Wolff, E. (1946) Les changements de sexe. Paris: Gallimard. 